\title{
Unraveling local spin polarization of Zhang-Rice singlet in lightly hole-doped cuprates using high-energy optical conductivity
}

\author{
Iman Santoso, ${ }^{1,2,12}$ Wei Ku, ${ }^{3, *}$ Tomonori Shirakawa, ${ }^{4,5,6}$ Gerd Neuber, ${ }^{7}$ Xinmao Yin, ${ }^{1,2}$ M. Enoki, ${ }^{8}$ Masaki Fujita, ${ }^{8}$ \\ Ruixing Liang, ${ }^{9}$ T. Venkatesan, ${ }^{1,10}$ George A. Sawatzky, ${ }^{9}$ Aleksei Kotlov, ${ }^{11}$ Seiji Yunoki, $, 4,5,6, \dagger$ \\ Michael Rübhausen, ${ }^{1,7}$ and Andrivo Rusydi ${ }^{1,2, \ddagger}$ \\ ${ }^{1}$ National University of Singapore Nanoscience and Nanotechnology Initiative (NUSNNI)-Nanocore, Department of Physics, \\ National University of Singapore, Singapore 117576 \\ ${ }^{2}$ Singapore Synchrotron Light Source, National University of Singapore, Singapore 117603 \\ ${ }^{3}$ Department of Physics and Astronomy, Shanghai Jiao Tong University, Shanghai 200240, China \\ ${ }^{4}$ Computational Condensed Matter Physics Laboratory, RIKEN, Wako, Saitama 351-0198, Japan \\ ${ }^{5}$ Computational Quantum Matter Research Team, RIKEN Center for Emergent Matter Science (CEMS), Saitama 351-0198, Japan \\ ${ }^{6}$ Computational Materials Science Research Team, RIKEN Advanced Institute for Computational Science (AICS), \\ Kobe, Hyogo 650-0047, Japan \\ ${ }^{7}$ Institut für Nanostruktur und Festkörperforschung, University of Hamburg, Luruper Chausse 149, Center for Free Electron Laser Science \\ (CFEL), D-22607 Hamburg, Germany \\ ${ }^{8}$ Institute for Material Research, Tohoku University, Katahira, Sendai 980-8577, Japan \\ ${ }^{9}$ Department of Physics and Astronomy, University of British Columbia, Vancouver, British Columbia, Canada V6T-1Z1 \\ ${ }^{10}$ Department of Electrical and Computer Engineering, National University of Singapore, Singapore 117576, Singapore \\ ${ }^{11}$ Photon Science Division, Deutsches Elektronensynchrotron (DESY), Notkestrasse 85, 22607 Hamburg, Germany \\ ${ }^{12}$ Departemen Fisika Fakultas Matematika dan Ilmu Pengetahuan Alam (FMIPA) Universitas Gadjah Mada, Sekip Utara Bulaksumur BLS 21, \\ Yogyakarta 55281, Indonesia \\ (Received 7 September 2015; revised manuscript received 29 December 2016; published 6 April 2017)
}

\begin{abstract}
Unrevealing local magnetic and electronic correlations in the vicinity of charge carriers is crucial in order to understand rich physical properties in correlated electron systems. Here, using high-energy optical conductivity (up to $35 \mathrm{eV}$ ) as a function of temperature and polarization, we observe a surprisingly strong spin polarization of the local spin singlet with enhanced ferromagnetic correlations between $\mathrm{Cu}$ spins near the doped holes in lightly hole-doped $\mathrm{La}_{1.95} \mathrm{Sr}_{0.05} \mathrm{Cu}_{0.95} \mathrm{Zn}_{0.05} \mathrm{O}_{4}$. The changes of the local spin polarization manifest strongly in the temperature-dependent optical conductivity at $\sim 7.2 \mathrm{eV}$, with an anomaly at the magnetic stripe phase $(\sim 25 \mathrm{~K})$, accompanied by anomalous spectral-weight transfer in a broad energy range. Supported by theoretical calculations, we also assign high-energy optical transitions and their corresponding temperature dependence, particularly at $\sim 2.5, \sim 8.7, \sim 9.7, \sim 11.3$, and $\sim 21.8 \mathrm{eV}$. Our result shows the importance of a strong mixture of spin singlet and triplet states in hole-doped cuprates and demonstrates a new strategy to probe local magnetic correlations using high-energy optical conductivity in correlated electron systems.
\end{abstract}

DOI: 10.1103/PhysRevB.95.165108

\section{INTRODUCTION}

As a prominent ingredient of the electronic and spin structures and of fundamental relevance for high-temperature superconductivity in copper oxides (cuprates), an understanding of magnetic and electronic correlations in relation to the charge carriers, i.e. doped holes, in their unusual normal state plays an important role. This is supported the fact that the superconductivity is induced by carriers doped into the antiferromagnetic insulator [1-6]. After nearly three decades of intensive studies, there is a general consensus that, in the cuprates, the doped holes go mainly into $\mathrm{O} 2 p$ orbitals. This has been experimentally observed by a number of experimental techniques, including x-ray absorption and energy-loss spectroscopies [7-10], angle-resolved photoelectron spectroscopy [11], inelastic neutron scattering [12], and

\footnotetext{
*weiku@mailaps.org

†yunoki@ riken.jp

${ }^{\ddagger}$ phyandri@nus.edu.sg
}

resonance sound velocity measurement [13]. However, how magnetic correlations evolved in the vicinity of doped holes remains a serious issue in the field.

Several theoretical models have suggested the importance of the oxygen sites [2,3,14-16]. However, local descriptions on how the doped hole correlated with the surrounding $\mathrm{Cu}$ spins remain hotly debated among different theoretical models. On one hand, a local singlet character of the doped hole in the $\mathrm{CuO}_{2}$ planes, the so-called Zhang-Rice singlet (ZRS) [2] was proposed. It consists of a doped hole on oxygen and an intrinsic local hole on $\mathrm{Cu}^{2+}$ in a singlet wave function with a net zero spin moment. On the other hand, a model of a three-spin polaron described that the doped hole in oxygen can promote local ferromagnetic fluctuations of $\mathrm{Cu}^{2+}$ spins surrounding it in an otherwise antiferromagnetic background $[3,17]$. Therefore, an experimental method that is capable of probing local magnetic correlations in the vicinity of charge carriers is needed to solve this fundamental problem.

The key challenge is to understand the nature of magnetic correlations in lightly hole-doped, high-quality single crystal cuprates even in the proximity of the antiferromagnetic regime 
[18]. Experimentally, because of the small concentration $(\sim 5 \%)$ of the doped holes, until now it has been very challenging to probe the short-range magnetic correlation around the small number of doped holes at the oxygen site, given the overwhelming contribution from the localized $\mathrm{Cu}$ spins.

\section{EXPERIMENTAL AND THEORETICAL METHODS}

Here, we design an experimental approach to address this fundamental problem. A combination of a synchrotron-based experimental technique, i.e. ultraviolet-vacuum ultraviolet (UV-VUV) optical reflectivity and spectroscopic ellipsometry is used to reveal the optical conductivity $\left(\sigma_{1}\right)$ of cuprates in an unprecedented energy range up to $32.5 \mathrm{eV}$, with very high accuracy, as a function of temperature and polarization. Note that the high-energy optical conductivity is sensitive to the magnetization as experimentally and theoretically shown in the case of manganites [19,20]. This novel technique is applied to high-quality untwinned single crystal of lightly doped, nonsuperconducting $\mathrm{La}_{1.95} \mathrm{Sr}_{0.05} \mathrm{Cu}_{0.95} \mathrm{Zn}_{0.05} \mathrm{O}_{4}$ ( $\mathrm{Zn}$ LSCO), and undoped $\mathrm{Sr}_{2} \mathrm{CuO}_{2} \mathrm{Cl}_{2}$ (SCOC), as a comparison, which were grown by the traveling solvent floating zone method. The Zn-LSCO has been characterized using neutron scattering revealing that our sample still shows the diagonal incommensurate spin modulation (or diagonal stripe phase) below $\sim 25 \mathrm{~K}\left(T_{s}\right)$ and the role of $\mathrm{Zn}$ was found to enhance the magnetic correlations [21]. This physical property is particularly important because (1) the antiferromagnetic background is still present in the excess of charge carriers, and (2) there exists a stripe phase below $T_{s}$ that demonstrates different local magnetic correlations surrounding the doped hole. The SCOC is isostructural of Zn-LSCO and regarded as a reference system for undoped cuprates [22]. Our analysis and assignments of optical transitions are supported with exact cluster diagonalizations and first-principles density functional calculations.

The normalization procedure of the reflectivity data is the following:

(1) Data collection and simulation: We collect and combine three sets of data: (1) $\Psi$ and $\Delta$ from spectroscopic ellipsometry with energy from 0.5 to $6 \mathrm{eV}$, (2) reflectivity $(R)$ from UV-VUV reflectance with energy from 3.5 to $35 \mathrm{eV}$, and (1) calculated form factors with energy from $30 \mathrm{eV}$ and well above taken from the Henke table [23]. The first two are from our experiments, while the latter was from a calculated value.

(2) Spectroscopic ellipsometry: Spectroscopic ellipsometry measures accurately the ellipsometric parameters $\Psi$ (the ratio between the amplitude of $p$ - and $s$-polarized reflected light) and $\Delta$ (represents the phase difference between $p$ and $s$-polarized reflected light) from which we then calculate reflectivity $(R)$, complex dielectric response, and optical conductivity accurately in the energy range of 0.5 to $6 \mathrm{eV}$ without the need of Kramers-Kronig transformation.

(3) The Henke tabulated value: From the table, one can extract the index of refraction (and the atomic form factor) in a very broad energy range, from 30 to $30000 \mathrm{eV}$. From here, we can calculate reflectivity of a material in that broad energy range.
(4) Normalization: We then normalize the UV-VUV reflectance spectra to reflectivity from the spectroscopic ellipsometry for the low-energy side and calculated reflectivity from the Henke tabulated value for the high-energy side. Therefore, we get the absolute value of the reflectivity from 0.5 to $35 \mathrm{eV}$. We note that the overlapping in the reflectivity spectrum at the low as well as high energies helps in our normalization procedure.

(5) Fitting: We then fit the comprehensive reflectivity data using the Lorentz oscillator model that fulfills the Kramers-Kronig transformation. During the fitting procedure, we shall be able to reproduce the results from spectroscopic ellipsometry, e.g. reflectivity, dielectric response, and optical conductivity. This procedure also validates our normalization procedure.

We perform both theoretical calculations and firstprinciples calculations using local spin density approximation $(\mathrm{LSDA}+\mathrm{U})$ and cluster calculations. The theoretical firstprinciples calculations using LSDA $+\mathrm{U}$ are performed for the $\mathrm{La}_{2} \mathrm{CuO}_{4}$ system, from which we extract total and partial density of states (DOS) associated with different atomic orbitals, i.e. $\mathrm{Cu}(1)$ and $\mathrm{Cu}(2)$ refer to two spin antiparallel $\mathrm{Cu}$ atoms in the system, $\mathrm{O}(1)$ the $\mathrm{O}$ atom in the $\mathrm{CuO}_{2}$ plane, and $\mathrm{O}(3)$ the apical oxygen out of the plane.

The details of cluster calculations are the following. The simplest model to capture the main excitations is a single $\mathrm{CuO}_{4}$ cluster, which includes five $3 d$ orbitals $\left(d_{x^{2}-y^{2}}, d_{z^{2}-r^{2}}, d_{x y}, d_{x z}\right.$, and $\left.d_{y z}\right)$ of copper and three $2 p$ orbitals $\left(p_{x}, p_{y}, p_{z}\right)$ of each oxygen. The undoped case is, thus, described by the $\mathrm{CuO}_{4}$ cluster with a single hole, and the additional hole doping simply increases the number of holes in the cluster. The cluster model used in this analysis is defined by the following Hamiltonian:

$$
\begin{gathered}
H=H_{\mathrm{pot}}+H_{t, p d}+H_{t, p p}+H_{U}, \\
H_{\mathrm{pot}}=\sum_{s, \alpha} E_{\alpha}^{d} d_{\alpha, s}^{\dagger} d_{\alpha, s}+\sum_{i, \beta, s} E_{i, \beta}^{p} p_{i, \beta, s}^{\dagger} p_{i, \beta, s}, \\
H_{t, p d}=t_{p d, \sigma} \sum_{s}\left[d_{b_{1 g}}, s^{\dagger}\left(-p_{1, x, s}+p_{2, y, s}\right.\right. \\
\left.\left.+p_{3, x, s}-p_{4, y, s}\right)+ \text { h.c. }\right]
\end{gathered}
$$

TABLE I. The parameter set used is listed. Here, $(p d \sigma)[(p d \pi)]$ is the Slater-Koster parameter for $\sigma(\pi)$ bonding between $p$ and $d$ orbitals [36]. These parameters are taken to match our experimental data.

\begin{tabular}{lccc}
\hline \hline Parameter & Value $(\mathrm{eV})$ & Parameter & Value $(\mathrm{eV})$ \\
\hline$t_{p d \sigma}[=\sqrt{3}(p d \sigma) / 2]$ & 1.3 & $E_{p \pi}$ & 2.0 \\
$t_{p d \pi}[=(p d \pi)]$ & -0.7 & $E_{p z}$ & 2.7 \\
$t_{p d z}[=(p d \sigma) / 2]$ & 0.65 & $U$ & 8.8 \\
$t_{p p \sigma}$ & -1.0 & $U^{\prime}$ & 6.5 \\
$t_{p p \pi}$ & 0.3 & $J$ & 1.2 \\
$E_{b_{1 g}}, E_{a_{1 g}}$ & 0 & $J^{\prime}$ & 1.2 \\
$E_{b_{2 g}}, E_{e_{g}}$ & 0.9 & $U_{p}$ & 4.0 \\
$E_{p \sigma}$ & 3.0 & $V_{p d}$ & 1.2 \\
\hline \hline
\end{tabular}



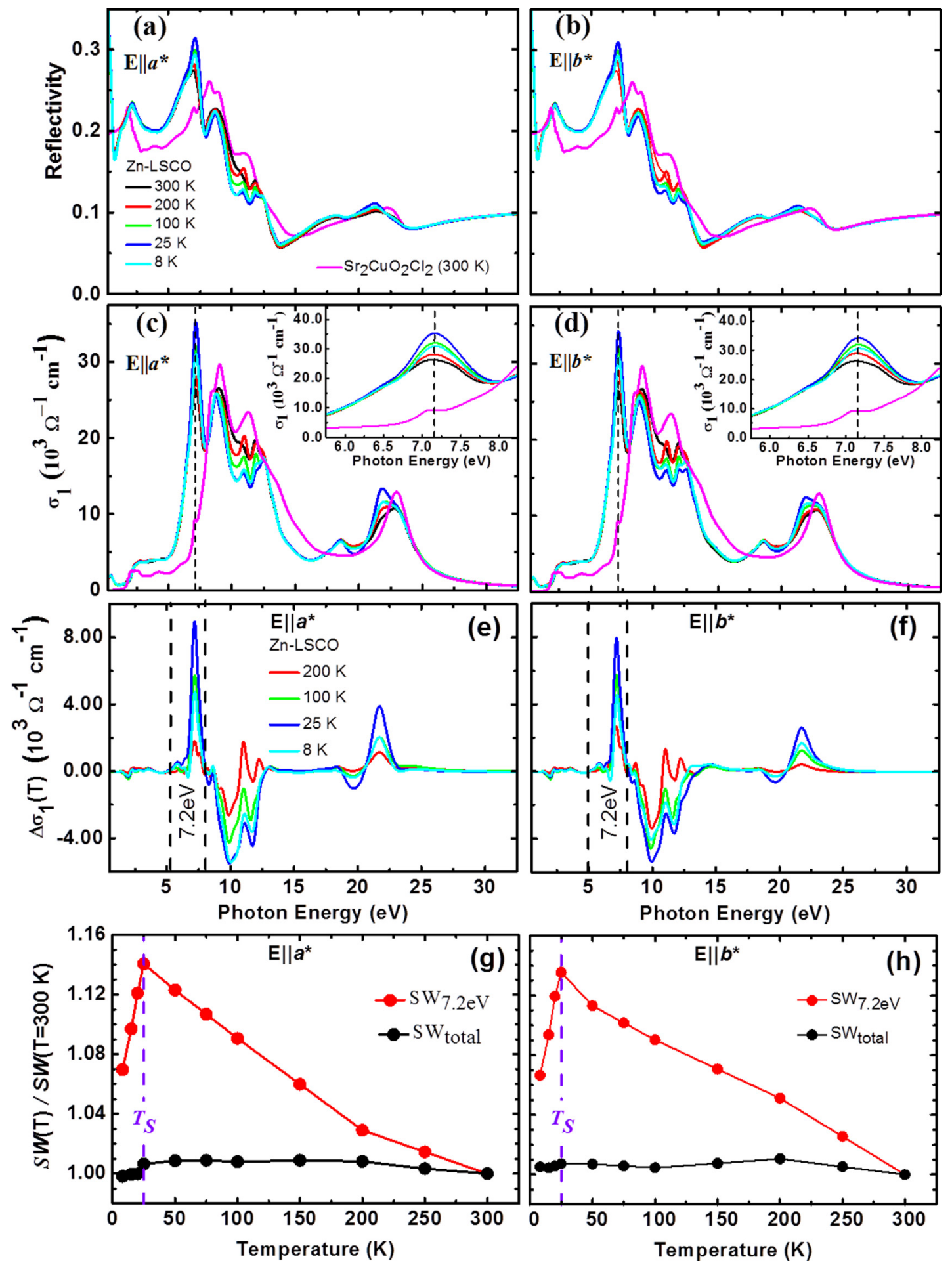

FIG. 1. (a) and (b) Reflectivity and (c) and (d) optical conductivity $\sigma_{1}$ of lightly hole-doped Zn-LSCO as a function of temperature and light polarizations as indicated in the figures. The undoped SCOC is used for comparison. The vertical-dashed lines show the new excitations at $7.2 \mathrm{eV}$. The inset of (a) shows the $\mathrm{CuO}$ plane with $\mathrm{CuO}_{4}$ plaquettes in the orthorhombic $a^{*}$ and $b^{*}$ axes. The inset of (c) and (d) is a magnification of excitations near $7.2 \mathrm{eV}$. (e) and (f) The change of optical conductivity $\Delta \sigma_{1}(T)$ and (g) and (h) relative changes of integrated SW. The $\Delta \sigma_{1}(T)$ is defined as $\Delta \sigma_{1}(T)-\Delta \sigma_{1}(T=300 \mathrm{~K})$. The relative change of the integrated $\mathrm{SW} \frac{\mathrm{SW}(T)}{\operatorname{SW}(300 \mathrm{~K})}$ is defined as $\frac{\int_{\omega_{1}}^{\omega_{2}} \Delta \sigma_{1}(\omega, T) d \omega}{\int_{\omega_{1}}^{\omega_{2}} \Delta \sigma_{1}(\omega, T=300 \mathrm{~K}) d \omega}$, where $T$ is temperature and $\omega_{1}\left(\omega_{2}\right)$ is the photon energy at $\omega_{1}\left(\omega_{2}\right)$. The integrated SW for two different regions $\mathrm{SW}_{\mathrm{II}}\left[\left(\omega_{1}\right.\right.$ to $\left.\omega_{2}\right)=($ from5.0to $\left.7.8 \mathrm{eV})\right]$ or as $\mathrm{SW}_{7.2 \mathrm{eV}}$, and $\mathrm{SW}_{\text {total }}$ (from 0.5 to $32.5 \mathrm{eV}$ ) are shown. The critical temperature for the diagonal stripe order is indicated by $T_{s}=25 \mathrm{~K}$. The overall $\mathrm{SW}(T)$ from 0.5 to $32.5 \mathrm{eV}$ is conserved within $0.2 \%$. 


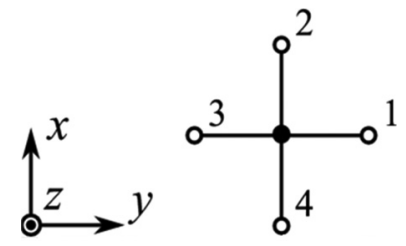

FIG. 2. Schematic figure of a $\mathrm{CuO}_{4}$ cluster. Black and white circles indicate copper and oxygen, respectively.

$$
\begin{aligned}
& +t_{p d, z} \sum_{s}\left[d _ { a _ { 1 g } , s } ^ { \dagger } \left(-p_{1, x, s}-p_{2, y, s}\right.\right. \\
& \left.\left.+p_{3, x, s}+p_{4, y, s}\right)+ \text { h.c. }\right] \\
& +t_{p d, \pi} \sum_{s}\left[d _ { b _ { 2 g } , s } ^ { \dagger } \left(p_{1, y, s}+p_{2, x, s}\right.\right. \\
& \left.\left.-p_{3, y, s}-p_{4, x, s}\right)+ \text { h.c. }\right] \\
& +t_{p d, \pi} \sum_{s}\left[d_{e_{g x}, s}^{\dagger}\left(p_{1, z, s}-p_{3, z, s}\right)+\text { h.c. }\right] \\
& +t_{p d, \pi} \sum_{s}\left[d_{e_{g y}, s}^{\dagger}\left(p_{2, z, s}-p_{4, z, s}\right)+\text { h.c. }\right],
\end{aligned}
$$

$$
\begin{aligned}
H_{t, p p}= & \frac{t_{p p, \sigma}}{2} \sum_{i=1}^{4} \sum_{s}\left\{\left[p_{i+1, x, s}^{\dagger}+(-1)^{i} p_{i+1, y, s}^{\dagger}\right]\right. \\
& \left.\times\left[p_{i, x, s}+(-1)^{i} p_{i, y, s}\right]+\text { h.c. }\right\} \\
& -\frac{t_{p p, \pi}}{2} \sum_{i=1}^{4} \sum_{s}\left\{\left[p_{i+1, x, s}^{\dagger}+(-1)^{i+1} p_{i+1, y, s}^{\dagger}\right]\right. \\
H_{U}= & \frac{U}{2} \sum_{\alpha, s} n_{d, \alpha, s} n_{d, \alpha, \bar{s}}+\frac{U^{\prime}-J}{2} \sum_{\alpha \neq \alpha^{\prime}, s} n_{d, \alpha, s} n_{d, \alpha^{\prime}, s} \\
+ & \frac{U^{\prime}}{2} \sum_{\alpha \neq \alpha^{\prime}, s} n_{d, \alpha, s} n_{d, \alpha^{\prime}, \bar{s}}-\frac{J}{2} \sum_{\alpha \neq \alpha^{\prime}, s} d_{\alpha, s}^{\dagger} d_{\alpha, \bar{s}} d_{\alpha, \bar{s}}^{\dagger} d_{\alpha^{\prime}, s} \\
+ & \frac{J}{2} \sum_{\alpha \neq \alpha^{\prime}, s} d_{\alpha, s}^{\dagger} d_{\alpha, \bar{s}}^{\dagger} d_{\alpha^{\prime}, \bar{s}} d_{\alpha^{\prime}, s} \\
+ & \frac{U_{p}}{2} \sum_{i, \beta, \beta^{\prime}, s, s^{\prime}} p_{i, \beta, s}^{\dagger} p_{i, \beta, s} p_{i, \beta^{\prime}, s^{\prime}}^{\dagger} p_{i, \beta^{\prime}, s^{\prime}} \\
+ & V_{p d} \sum_{i} \sum_{\alpha, \beta, s, s^{\prime}} d_{\alpha, s}^{\dagger} d_{\alpha, s} p_{i, \beta, s}^{\dagger} p_{i, \beta, s},
\end{aligned}
$$

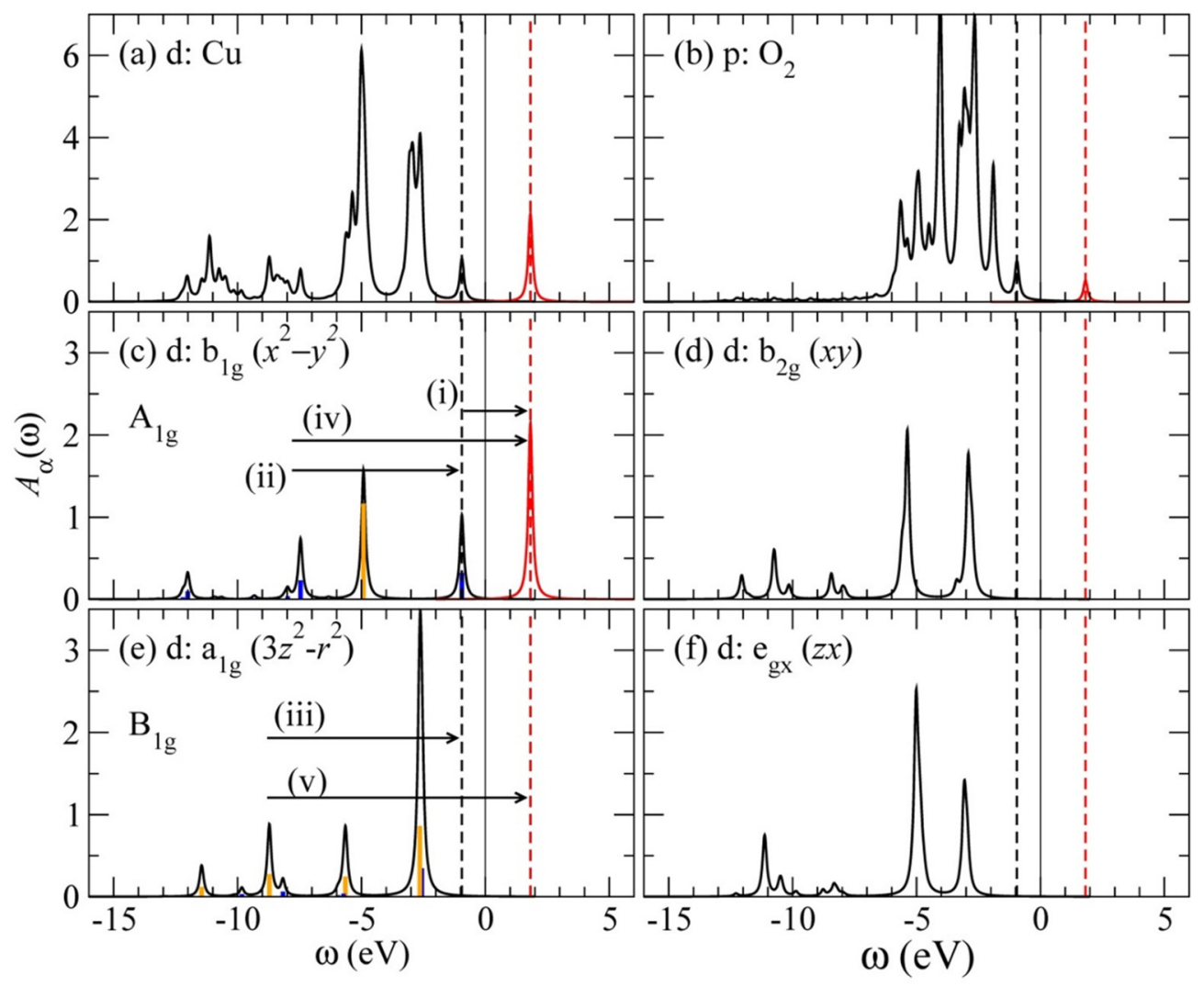

FIG. 3. Projected one-electron removal and one-electron addition spectral functions $A_{\alpha}(\omega)$ for the undoped $\mathrm{CuO}_{4}$ cluster. The symmetry $\Gamma_{\alpha}$ (in $D_{4 h}$ ) for orbital $\alpha$ is indicated in the figures (c)-(f). Since the ground state of the undoped system is of $b_{1 g}$ symmetry, the symmetry of the one-electron removal (addition) states is easily obtained by $\Gamma_{\alpha} \times b_{1 g}$. The total symmetries for the electron removal states of (c) $A_{1 g}$ and (e) $B_{1 g}$ represent the two hole states of current interest. Orange and blue lines indicate triplet and singlet states, respectively. For comparison, the one-electron removal spectra for all (a) $\mathrm{Cu} 3 d$ and (b) $\mathrm{O} 2 p$ orbitals are also shown. The states below zero (the Fermi energy indicated by solid lines) are the electron removal states, and those above zero are the electron addition states. The ZRS (UHB) is indicated by black (red) dashed lines. 

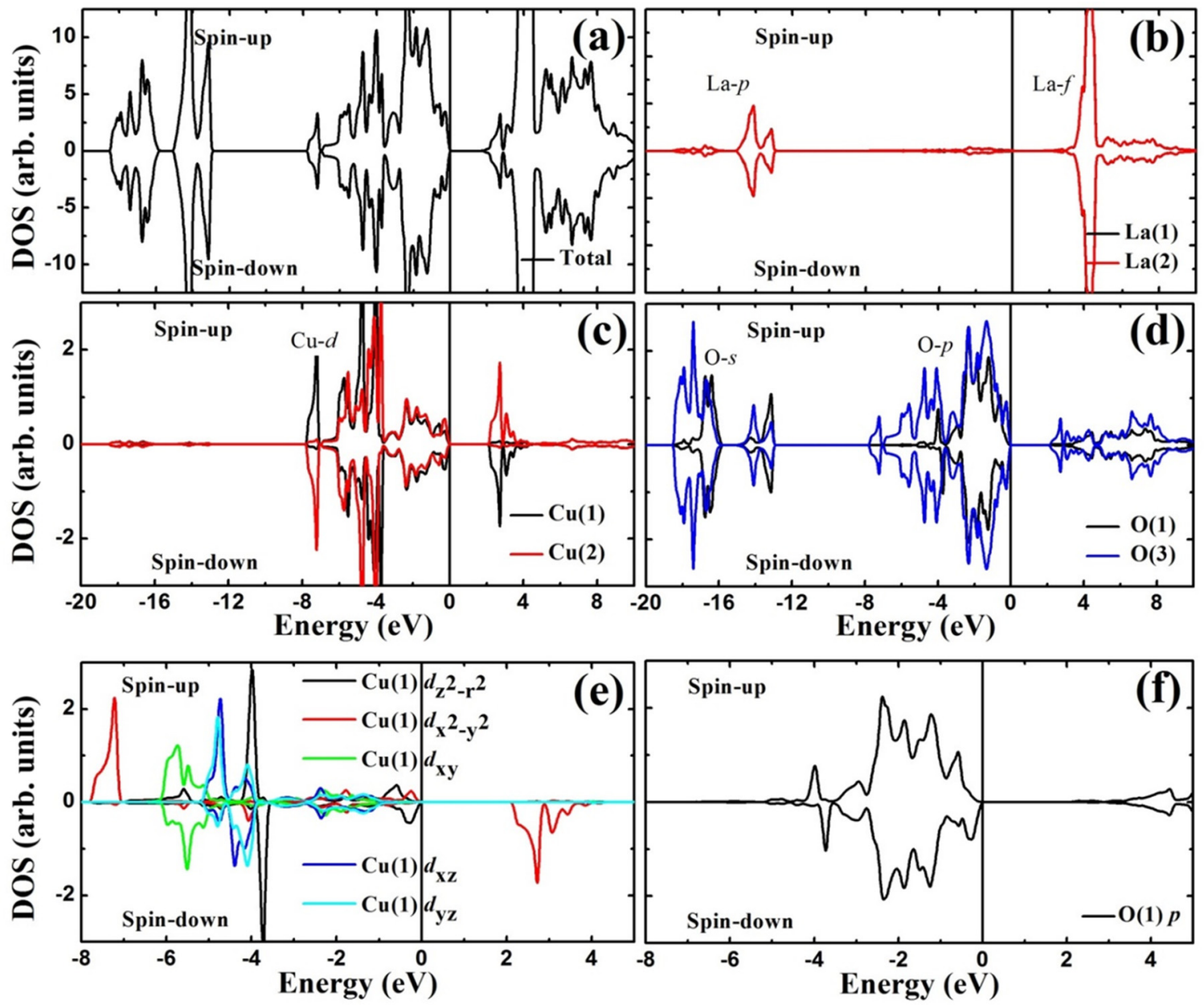

FIG. 4. Theoretical first-principles calculations using LSDA + U for parent $\mathrm{La}_{2} \mathrm{CuO}_{4}$ system. (a) Total and (b)-(f) partial DOS associated with different atomic orbitals. $\mathrm{Cu}(1)$ and $\mathrm{Cu}(2)$ refer to two spin antiparallel $\mathrm{Cu}$ atoms in the system, $\mathrm{O}(1)$ the $\mathrm{O}$ atom in the $\mathrm{CuO}$, plane, and $\mathrm{O}(3)$ the apical oxygen out of the plane. The energy range of the plot are (a)-(d) -32.5 to $17.5 \mathrm{eV}$ and (e)-(f) $-8 \mathrm{eto} 5 \mathrm{eV}$.

where $d_{\alpha, s}^{\dagger}\left(d_{\alpha, s}\right)$ creates (annihilates) a hole with orbital $\alpha\left(\alpha=b_{1 g}, a_{1 g}, b_{2 g}, e_{g x}, e_{g y}\right)$ and spin $\left(s= \pm \frac{1}{2}\right)$ on copper, and $n_{d, \alpha, s}=d_{\alpha, s}^{\dagger} d_{\alpha, s}$. Note that $b_{1 g}, a_{1 g}, b_{2 g}, e_{g x}$, and $e_{g y}$ correspond to $d_{x^{2}-y^{2}}, d_{3 z^{2}-r^{2}}, d_{x y}, d_{x z}$, and $d_{y z}$ orbitals, respectively, which form the bases of the irreducible representations of point group symmetry $D_{4 h}$. Here, $p_{i, \alpha, s}^{\dagger}\left(p_{i, \alpha, s}\right)$ is the creation (annihilation) operator of a hole at the $i$ th oxygen with $\alpha(=x, y, z)$ orbital and spin $s$. Here, we impose that $p_{5, \alpha, s}^{\dagger}=p_{1, \alpha, s}^{\dagger}$. Also, $H_{\text {pot }}$ is the onsite potential energy term with $E_{1, y}^{p}=E_{2, x}^{p}=$ $E_{3, y}^{p}=E_{4, x}^{p}=E_{p \pi}^{p}$ and $E_{i, z}^{p}=E_{p z}^{p}$, due to the crystal-field effect. Additionally, $H_{t, p d}$ and $H_{t, p p}$ are the hopping terms for the nearest neighbor $\mathrm{Cu}-\mathrm{O}$ bonds and the nearest neighbor $\mathrm{O}-\mathrm{O}$ bonds, respectively. Here, $H_{U}$ is the interaction term including the onsite intraorbital Coulomb repulsion $U$ on the copper, the onsite interorbital Coulomb repulsion $U^{\prime}$, the Hund's coupling $J$, the pair hopping term $J^{\prime}$ at the $\mathrm{Cu}$ site, the onsite Coulomb interaction $U_{p}$ at the $\mathrm{O}$ site, and the nearest neighbor Coulomb interaction $V_{p d}$ between the $\mathrm{Cu}$ and $\mathrm{O}$ sites. The parameters used in this paper are shown in Table I. Note that these parameters are matched with our experimental results and are consistent with those reported by previous papers $[15,24]$.

\section{RESULTS AND DISCUSSION}

In Fig. 1, we show high-energy reflectivity [Figs. 1(a) and 1(b)] and high-energy optical conductivity $\sigma_{1}$ [Figs. 1(c) and 1(d)] of Zn-LSCO as a function of temperature (from 8 to $300 \mathrm{~K}$ ) and polarization $\left[\mathbf{E} \| \boldsymbol{a}^{*}\right.$ and $\mathbf{E} \| \boldsymbol{b}^{*}$, see inset of Fig. 1(a)], together with $\mathrm{SCOC}$ for $\mathbf{E} \perp \boldsymbol{c}$ as a reference. Intriguingly, new optical transitions are observed at high-energies $(>3 \mathrm{eV})$, i.e. a pronounced peak at $7.2 \mathrm{eV}$ and a rather weak peak at $21.8 \mathrm{eV}$. Supported by theoretical calculations, these new peaks originate from excitations involving doped holes in the oxygen orbitals of the $\mathrm{Cu}-\mathrm{O}$ planes; they only occur in Zn-LSCO, and they are absent in the undoped reference sample even though the overall spectrum of the optical conductivity is very similar, showing that it is dominated by the copper-oxygen planes. Furthermore, we also observe other high-energy optical transitions, e.g. at $\sim 8.7, \sim 9.7$, and $\sim 11.3 \mathrm{eV}$, which occur in both samples but with some smaller differences in their details. At low energies 
(a)

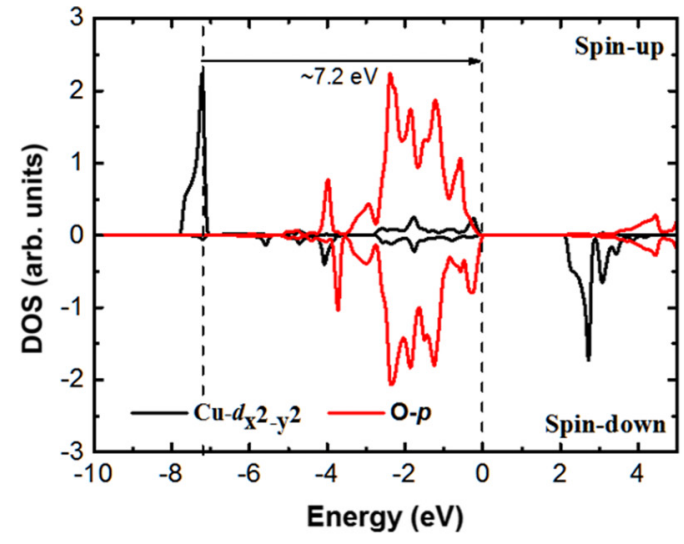

(b)

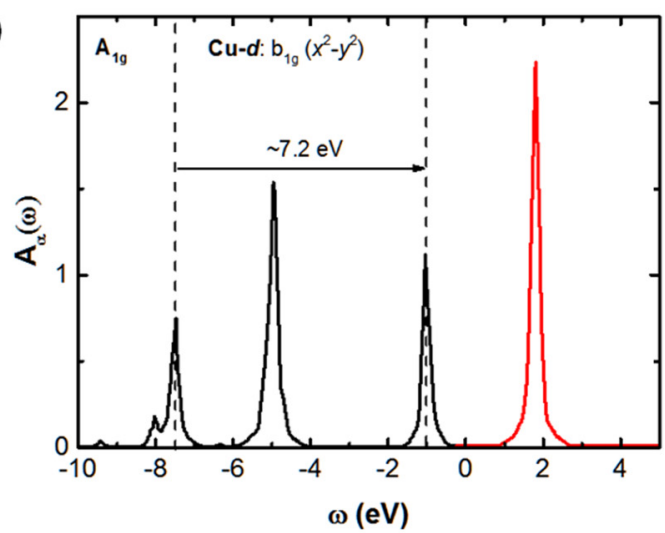

(c)

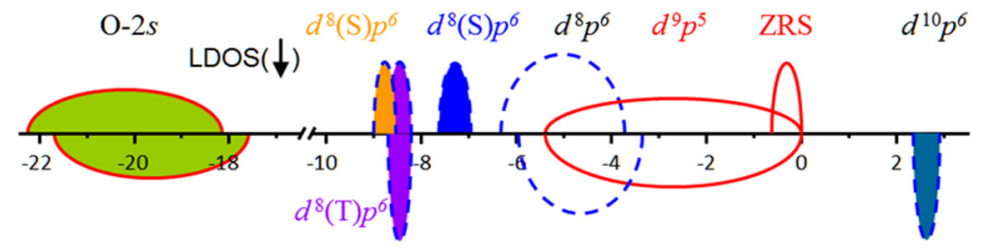

(d)

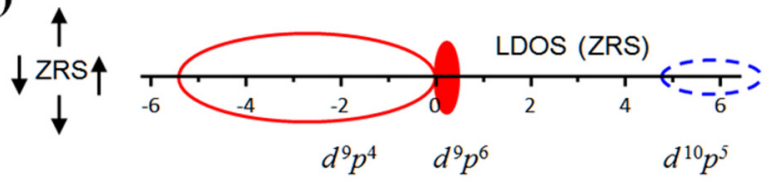

Unpolarized: stand-alone zhang-rice singlet (ZRS)

(e)

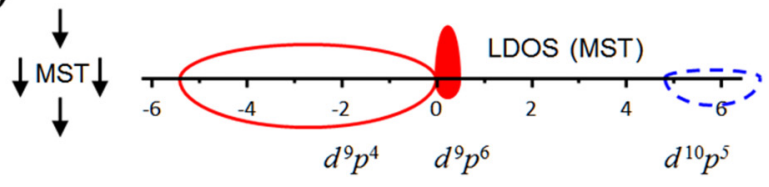

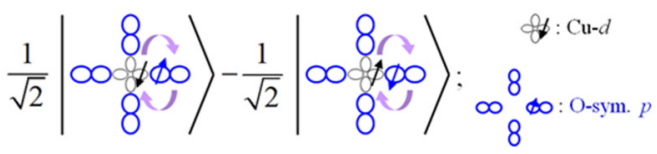

Polarized by the surrounding spins: Mixed singlet and triplet (MST)

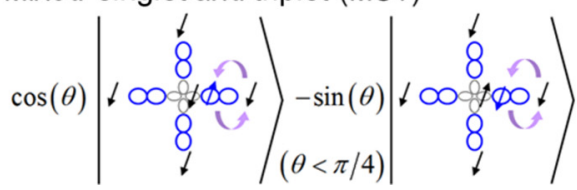

FIG. 5. (a) DOS based on LSDA + $\mathrm{U}$ for $\mathrm{Cu} d_{x^{2}-y^{2}}$ and $\mathrm{O} p$ of the parent compound $\mathrm{La}_{2} \mathrm{CuO}_{4}$ system. (b) Projected one-electron removal and one-electron addition spectral functions $A_{\alpha}(\omega)$ for the undoped $\mathrm{CuO}_{4}$ cluster and the total symmetries for the electron removal states of $A_{1 g}$. The symmetry $\Gamma_{\alpha}$ (in $D_{4 h}$ ) for orbital $\alpha$ is indicated. Since the ground state of the undoped system is of $b_{1 g}$ symmetry, the symmetry of the one-electron removal (addition) states is obtained by $\Gamma_{\alpha} \times b_{1 g}$. The total symmetries for the electron removal states of $A_{1 g}$ represents the two hole states of current interest. (c) Conceptual local DOS (LDOS) based on experimental result and calculations with local spin density approximations for the undoped case. Acronyms $\mathrm{S}$ and $\mathrm{T}$ stand for singlet and triplet states, respectively. Here, $d^{n}\left(p^{n}\right)$ indicates the number $n$ of $d(p)$ electrons in $\mathrm{Cu}(\mathrm{O})$. The multiple level in the $\mathrm{Cu} d^{8}\left[d^{8}(\mathrm{~S}) p^{6}\right]$ singlet configuration is due to the crystal field splitting. (We note that the same symbols are used in Figs. 3 and 4). (Left) Spin structure, (middle) LDOS, and (right) local wave function for (d) unpolarized, standalone ZRS, and (e) strongly spin polarized of MST. LDOS ( $\downarrow)$ denotes spin down. Note that the same symbols are used in Fig. 3.

$(<3 \mathrm{eV})$, a well-known charge transfer gap is observed in both hole-doped $\mathrm{Zn}$-LSCO at $\sim 2.5 \mathrm{eV}$ and undoped SCOC at a slightly lower energy of $\sim 2.0 \mathrm{eV}$. However, only in the hole-doped Zn-LSCO, we observe a midinfrared response $(\sim 0.7 \mathrm{eV})$, resulting from the holes doped into the system [25].

The key observation of our measurement is the surprisingly strong temperature dependence of the $7.2 \mathrm{eV}$ peak as clearly shown in the change of optical conductivity $\Delta \sigma_{1}[($ Figs. 1(e) and $1(\mathrm{f})]$. We observe a spectral-weight (SW) transfer as high as $15 \%$ [Figs. $1(\mathrm{~g})$ and $1(\mathrm{~h})$ ] with an anomaly around $T_{s}$. As the temperature decreases, these peaks steadily enhance. Intriguingly, when the diagonal stripe phase develops at $T_{S}$ [21], an abrupt change occurs, and these peaks decrease dramatically as temperature further decreases. Such a new optical anomaly near the stripe phase is important to a model system as it reveal the local spin configurations surrounding the doped holes. Correspondingly, in accordance to the first-moment sum rule, a large temperature-dependent SW loss is observed in the energy range of 7.6-20.9 eV, which is three orders of magnitude higher than any thermal energy scale $(<\sim 30 \mathrm{meV})$. This strongly points toward strong electronic correlations. As an example, the bare Coulomb onsite repulsion in cuprates is of the order of $10-20 \mathrm{eV}$ [26]. The total integrated SW is conserved to within $0.2 \%$, which allows us to reveal magnetic and charge correlations in cuprates.

We perform exact cluster diagonalization and firstprinciples density functional calculations within LSDA $+\mathrm{U}$. We are mainly interested in the identification of the basic optical transitions which are mostly the intercluster excitations. 
(a)

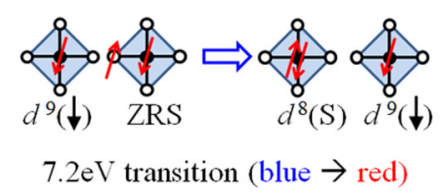

(b) Transitions to unpolarized ZRS
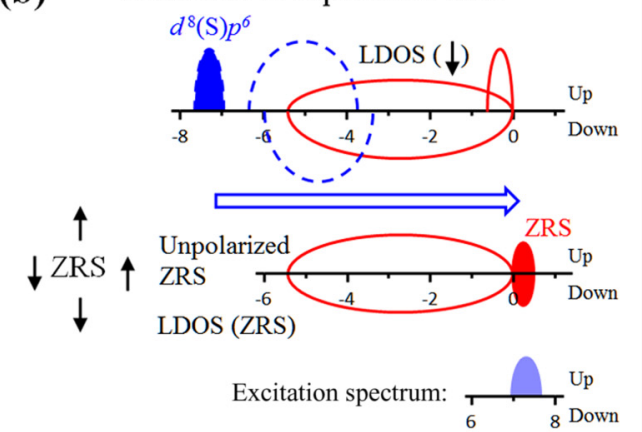

(c) Transitions to "polarized"MST

(d)
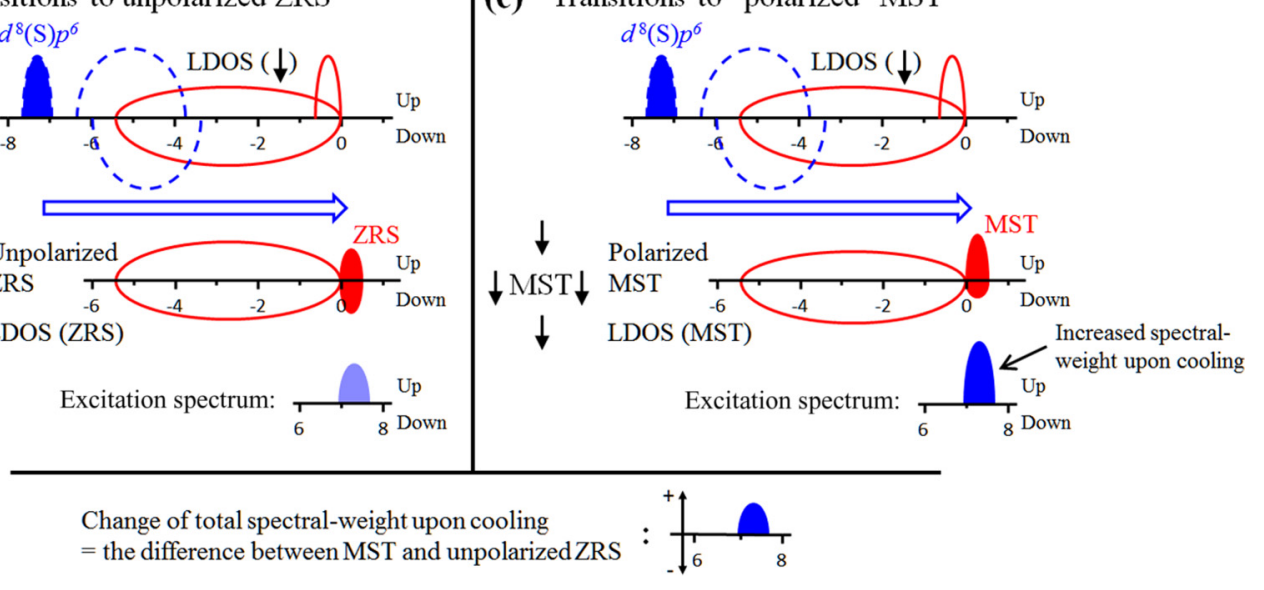

FIG. 6. (a) Schematic hole configurations of the $7.2 \mathrm{eV}$ optical transition within two $\mathrm{CuO}_{4}$ plaquettes. The left (right) two CuO $\mathrm{Claquettes}_{4}$ represent the initial (final) state of the corresponding optical excitation. The $d^{9}$ plaquette state corresponds to the ground state of the $\mathrm{CuO}_{4}$ plaquette with a single hole, and the ZRS plaquette corresponds to a state with two holes, one in $\mathrm{Cu}$ and one in $\mathrm{O}$ sites. The energy of the optical excitation is calculated from the $\mathrm{CuO}_{4}$ plaquette model, matched with experimental data. (b) An intersite optical transition and excitation spectrum of unpolarized ZRS corresponding to the $\mathrm{CuO}_{4}$ plaquettes configuration in (a). The optical transition occurs by exciting an electron from (top panel) $d^{8}(\mathrm{~S}) p^{6}$ state of $d^{9}(\downarrow) \mathrm{CuO}_{4}$ plaquette into (middle panel) the unpolarized $\mathrm{ZRS} \mathrm{CuO}_{4}$ plaquette. (Bottom panel) This optical transition is allowed only for spin-up configuration; therefore, the resulting excitation spectrum is for spin-up configuration. Such an optical transition does not change as a function of temperature because it does not depend on surrounding spin configurations. (c) An intersite optical transition and excitation spectrum of polarized MST. The optical transition occurs by exciting an electron from (top panel) $d^{8}(\mathrm{~S}) p^{6}$ state of $d^{9}(\downarrow) \mathrm{CuO}_{4}$ plaquette into (middle panel) the polarized $\mathrm{MST} \mathrm{CuO}_{4}$ plaquette. (Bottom panel) This optical transition is allowed only for spin-up configuration with a strong temperature dependence. As temperature decreases, such an optical excitation increases because the local ferromagnetic correlation enhances, consistent with experimental data. (d) The temperature dependence in the MST configuration yields a robust temperature-dependent optical transition at $7.2 \mathrm{eV}$ as temperature decreases, fully consistent with our experimental result (cf Fig. 1). LDOS $(\downarrow)$ denotes spin down of hole.

These are basically represented by the electron removal states of one cluster combined with the electron addition states of a neighboring cluster, which we consider to be uncoupled from each. Let us first calculate the one-electron removal and one-electron addition spectral functions $A_{\alpha}(\omega)=A_{\alpha}^{+}+A_{\alpha}^{-}$ for the undoped case. Here,

$$
A_{\alpha}^{+}(\omega)=-\frac{1}{\pi} \lim _{\delta \rightarrow 0^{+}} \operatorname{Im}\left\langle\psi_{0}\left|c_{\alpha}^{\dagger} \frac{1}{\omega-H+E_{0}+i \delta} c_{\alpha}\right| \psi_{0}\right\rangle,
$$

and

$$
A_{\alpha}^{-}(\omega)=-\frac{1}{\pi} \lim _{\delta \rightarrow 0^{+}} \operatorname{Im}\left\langle\psi_{0}\left|c_{\alpha} \frac{1}{\omega-E_{0}+H+i \delta} c_{\alpha}^{\dagger}\right| \psi_{0}\right\rangle,
$$

where $c_{\alpha}\left(=d_{\alpha}, p_{\beta}\right)$ corresponds to the annihilation operator of hole, and $\left|\psi_{0}\right\rangle$ is the ground state of the $\mathrm{CuO}_{4}$ cluster with a single hole (see Fig. 2). As shown in Fig. 3, all excitations are classified by using their symmetry. For instance, one particle electron-removal spectral function, corresponding to the electron removal of a $x^{2}-y^{2}$ symmetry electron resulting in two holes in $\mathrm{CuO}_{4}$, with $A_{1 g}$ symmetry are shown in Fig. 3(c).

Theoretical first-principles calculations using LSDA $+\mathrm{U}$ were performed for the $\mathrm{La}_{2} \mathrm{CuO}_{4}$ system, from which we extract total and partial DOS associated with different atomic orbitals, i.e. $\mathrm{Cu}(1)$ and $\mathrm{Cu}(2)$ refer to two spin antiparallel $\mathrm{Cu}$ atoms in the system, $\mathrm{O}(1)$ the $\mathrm{O}$ atom in the $\mathrm{CuO}_{2}$ plane, and $\mathrm{O}(3)$ the apical oxygen out of the plane, as shown in Fig. 4.

The resulting energies of different electronic states and their spin polarization for the local copper and oxygen sites are shown in Figs. 5(a) and 5(b), for an undoped cuprate. From this, it is clear that spin polarization is favored for $\mathrm{O}(1)$ states, suggesting a strong triplet contribution [cf Fig. 5(a)]. Consistent with cluster calculations as shown in Fig. 5(b), the main transition comes from the $\mathrm{Cu} d_{x^{2}-y^{2}}$, which is $\sim 7.2 \mathrm{eV}$ below the Fermi level. Based on the optical transition rule, the hole doping is then essential to activate this optical transition (see discussion below). Therefore, we would not expect to see this state in undoped but rather in slightly doped cuprates. Pictorial many-body schemes are shown in Figs. 2(c) and 2(d). Figure 2(c) describes a $\mathrm{Cu} d^{9}$ site with spin pointing up (one spin-down hole, as shown in the plot). In this configuration, the ZRS appears below the chemical potential as it is possible to create a ZRS by adding a spin-up hole to the oxygen site. Figure 5(d) describes a $\mathrm{Cu} d^{9}$ with a ligand hole in the ZRS configuration, so it is possible to destroy the ZRS by adding an electron (above the chemical potential) in either spin channel. 

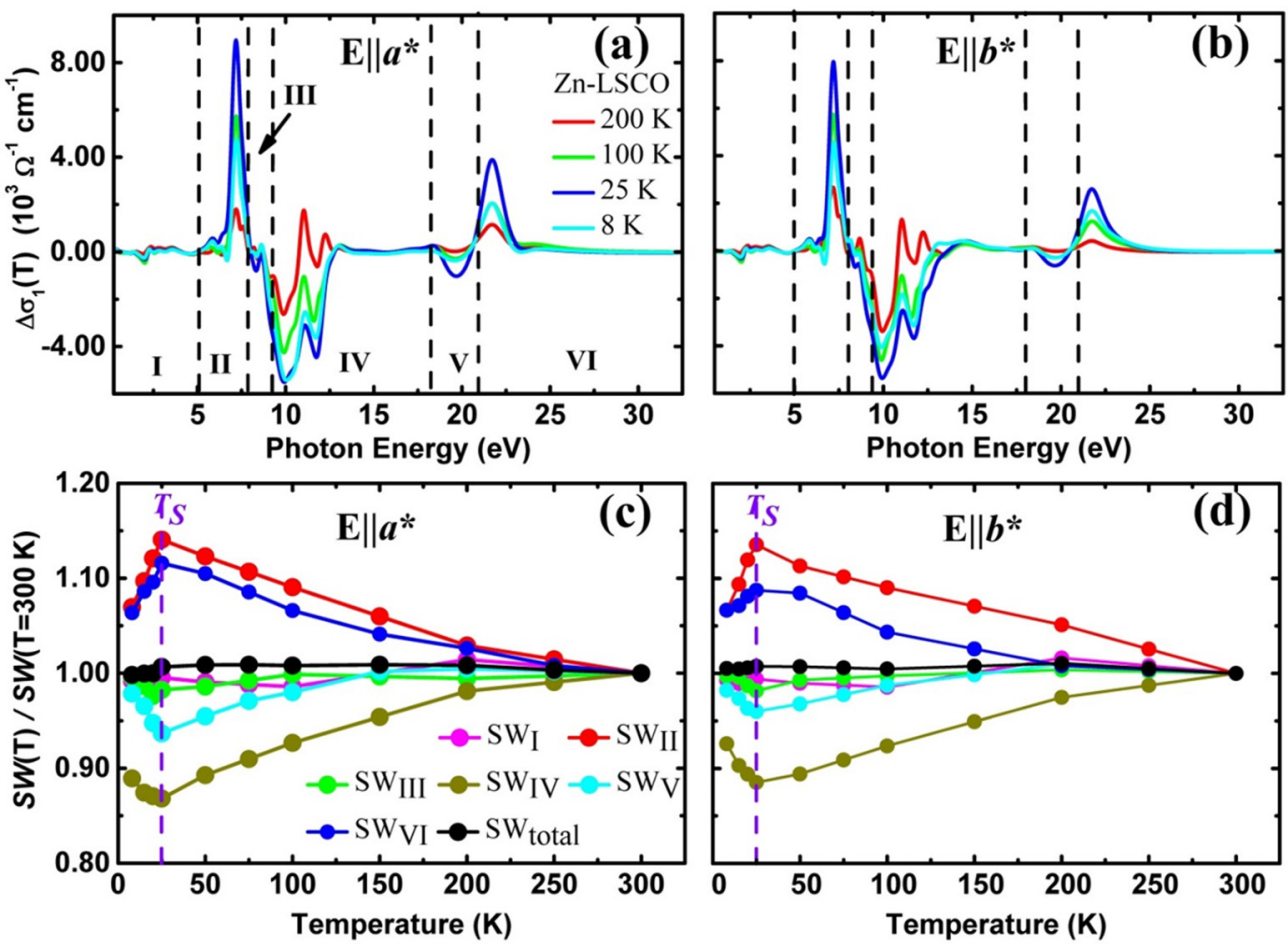

FIG. 7. SW analysis for different incoming light polarizations $E \|\left(a^{*}, b^{*}\right)$ and different spectral regions of Zn-LSCO. (a) and (b) Change of optical conductivity $\Delta \sigma_{1}(T)$ defined as $\sigma_{1}(T)-\sigma_{1}(T=300 \mathrm{~K})$ and (c) and (d) relative change of the integrated $\mathrm{SW} \frac{\mathrm{SW}(T)}{\mathrm{SW}(300 \mathrm{~K})}$ defined as $\frac{\int_{\omega_{1}}^{\omega_{2}} \Delta \sigma_{1}(\omega, T) d \omega}{\omega_{\omega_{1}} \Delta \sigma_{1}(\omega, T=300 \mathrm{~K}) d \omega}$, where $T$ is temperature $(\mathrm{K})$ and $\omega_{1}\left(\omega_{2}\right)$ is the photon energy at $\omega_{1}\left(\omega_{2}\right)$. We show the integrated SW for seven different regions $\mathrm{SW}_{\mathrm{I}}\left[\left(\omega_{1}\right.\right.$ to $\left.\omega_{2}\right)=$ (from 0.5 to $\left.\left.5.0 \mathrm{eV}\right)\right], \mathrm{SW}_{\mathrm{II}}$ (from 5.0 to $7.8 \mathrm{eV}$ ) or as $\mathrm{SW}_{7.2 \mathrm{ev}}$ in the main text, $\mathrm{SW}_{\mathrm{III}}\left(\right.$ from 7.8 to $9.2 \mathrm{eV}$ ), $\mathrm{SW}_{\mathrm{IV}}$ (from 9.2 to $18.0 \mathrm{eV}$ ), $\mathrm{SW}_{\mathrm{V}}$ (from 18.0 to $20.8 \mathrm{eV}$ ), $\mathrm{SW}_{\mathrm{VI}}$ (from 20.8 to $21.5 \mathrm{eV}$ ) or as $\mathrm{SW}_{21.8 \mathrm{ev}}$ in the main text, and $\mathrm{SW}_{\text {total }}($ from 0.5 to $32.5 \mathrm{eV})$. Here, $\Delta \sigma_{1}(T)$ and $\mathrm{SW}(T) / \mathrm{SW}(300 \mathrm{~K})$ for different polarization and spectral regions are indicated in the figures. The critical temperature for the diagonal stripe order is indicated by $T_{s}$ [21]. We focus our discussion on $\mathrm{SW}_{\text {III }}$ for explaining the $\sigma_{1}$ at $8.7 \mathrm{eV}$ and on $\mathrm{SW}$ IV for 9.7 and $11.3 \mathrm{eV}$. The overall $\mathrm{SW}(T)$ from 0.5 to $32.5 \mathrm{eV}$ is conserved within $0.2 \%$.

The high-energy optical conductivity involves high-energy states of the $\mathrm{Cu} d^{8}$ orbitals, which are well known to exist in the 7 to $15 \mathrm{eV}$ range (see also Refs. [27,28] for other cuprates) and can be understood via intersite transitions involving $\mathrm{O}$ and the neighboring two $\mathrm{Cu}$ sites. The most relevant microscopic processes of the transition at $7.2 \mathrm{eV}$, showing a strong temperature dependence, are illustrated in Fig. 6. They are dominated by transitions involving $\mathrm{O} 2 p$ orbitals and the $\sigma$-bonding $\mathrm{Cu} 3 d_{x^{2}-y^{2}}$ or $\mathrm{Cu} 3 d_{3 Z^{2}-r^{2}}$ orbitals. Since one of the holes on $\mathrm{Cu} d^{8}$ must be a $d_{x^{2}-y^{2}}$ hole, we consider only the $d^{8}$ states with two $d_{x^{2}-y^{2}}$ holes, which form spin singlets or one $d_{x^{2}-y^{2}}$ and one $d_{3 Z^{2}-r^{2}}$ hole, which can also form a triplet. In a pure singlet scenario, the $7.2 \mathrm{eV}$ peak should be independent from temperature because, in such a scenario, the peak originated largely from excitations of a $\mathrm{O} 2 p$ doped hole centered on one $\mathrm{Cu}$ (in a ZRS) to a neighboring $\mathrm{Cu} 3 d$ orbital forming a singlet state $d^{8}(S):\left|d^{9} \mathrm{ZRS}\right\rangle \rightarrow\left|d^{8}(S) d^{9}\right\rangle$ [see Fig. 6(b)], while other transitions, i.e. $\sim 8.7, \sim 9.7$, and $\sim 11.3 \mathrm{eV}$ and $21.8 \mathrm{eV}$ features, which are present at both Zn-LSCO and SCOC samples, are dominated by the copper-oxygen planes as discussed below.

The strong temperature dependence of the $7.2 \mathrm{eV}$ peak reveals an important and yet surprising nature of the ZRS state, namely the $\mathrm{Cu}$ and $\mathrm{O}$ orbitals being actually spin polarized rather than spin neutral as in a pure singlet form [see also Fig. 2(a)]. Note that, in strongly correlated materials, even spin-preserved charge response of electrons is still constrained by the spin correlation of the system due to the Pauli exclusion principle. Hence, the wave function has to contain now a mixed singlet and triplet (MST) wave function [Figs. 2(e) and 3(c)], instead of the pure singlet [Figs. 2(d) and 3(b)]. Since the local ferromagnetic correlations are expected to be enhanced as the temperature is being reduced until $T_{s}$, the temperature evolution of the $7.2 \mathrm{eV}$ peak is a direct example of this scenario [Fig. 3(c)]. Only the component of the wave function that would have ferromagnetically aligned $\mathrm{Cu}$ spins can contribute to the temperature-dependent optical transition matrix element [cf Figs. 3(c) and 3(d)]. Therefore, an increase of the SW of this excitation with decreasing temperature indicates an increase of a ferromagnetic correlation between the surrounding $\mathrm{Cu}$ spins, reflecting the influence of the $\mathrm{O} 2 p$ hole.

This somewhat unexpected conclusion can be understood from the consideration of the correlation between the MST and the neighboring $\mathrm{Cu}$ spins [Fig. 5(c)]. In the MST, the symmetry in the spin degree of freedom is broken because the surrounding $\mathrm{Cu}$ spins are strongly polarized in one direction. Consequently, the MST benefits from a ferromagnetically aligned component between neighboring $\mathrm{Cu}$ sites. This allows 
(a)

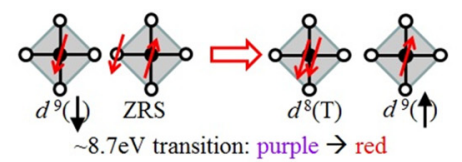

(b)

Transitions to unpolarized ZRS

(d)

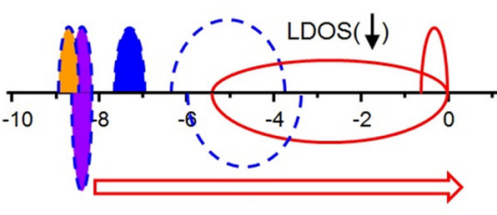

Unpolarized ZRS

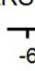

LDOS( ZRS )

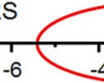

Excitation spectrum:

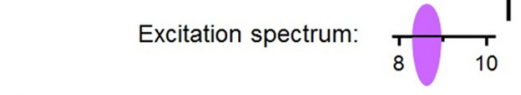

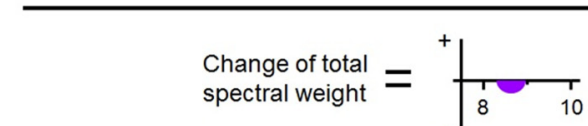

(c) Transitions to polarized mixed singlet and triplet (MST)

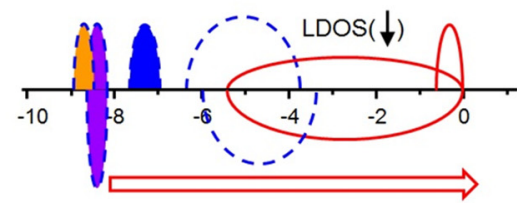

Polarized MST

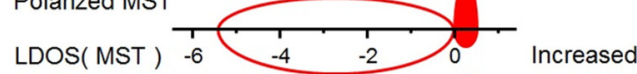

Excitation spectrum:

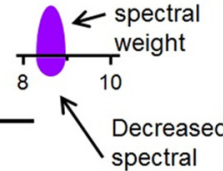

weight

FIG. 8. Pictorial model of the electronic band structure and optical transitions at $8.7 \mathrm{eV}$. (a) Schematic electronic and spin configurations of $\mathrm{CuO}_{4}$ plaquettes consisting of a doped hole forming a ZRS. In each set of figures, the left (right) two $\mathrm{CuO}_{4}$ plaquettes represent the initial (final) state of the corresponding optical excitation. The $d^{9}$ plaquette state corresponds to the ground state of the $\mathrm{CuO}_{4}$ plaquette with a single hole, and the ZRS plaquette corresponds to a state with two holes, one in $\mathrm{Cu}$ and one in $\mathrm{O}$ sites. Assuming each plaquette is independent, the optical excitation energy is easily estimated from the $\mathrm{CuO}_{4}$ plaquette model. Excitation spectrum for (b) transition to unpolarized ZRS and (c) transition to polarized MST [cf Figs. 6(b) and 6(c)]. LDOS ( $\downarrow$ ) and LDOS ( $\uparrow$ ) denote spin down and spin up, respectively. (d) Change of total $\mathrm{SW}$ as a function of temperatures expected from this model. Note that the more complete pictorial model of electronic band structure can be seen at Fig. 5(a).

maximizing the virtual kinetic processes [cf purple arrows Fig. 5(c)] between the doped hole at the $\mathrm{O}$ sites and the intrinsic hole in the neighboring $\mathrm{Cu}$ sites. Hence, the process leads effectively to a mixing of a triplet component into the wave function. This is the same microscopic process that leads to the formation of the three-spin polaron model in the O-centered local picture [16,17]. Obviously, the more the surrounding $\mathrm{Cu}$ spins align, the stronger this effect is, and the stronger the $7.2 \mathrm{eV}$ peak grows [cf inset of Figs. 1(c) and 1(d)] showing that the ferromagnetic alignment of the neighboring $\mathrm{Cu}$ spins seems to be the lowest energy state since the ferromagnetic correlations increase with decreasing temperatures.

Our measurement addresses an important issue on the lowtemperature magnetic structure at $T<T_{s}$. Interestingly, the new $7.2 \mathrm{eV}$ peak is also very sensitive to the formation of a long-range ordered stripe phase [21], i.e. the abrupt reversal of the trend in the SWs near $T_{s}$, which can be explained within the MST picture. The stripe correlation hosts an antiphase boundary of the antiferromagnetic correlation across the doped hole [29-34]. That is, the $\mathrm{Cu}$ atoms on the opposite side of the MST are correlated with the opposite spin. This leads to a compensation of the net magnetic moment of the surrounding spins of the MST and consequently to the observed abrupt decrease of intensity in the $7.2 \mathrm{eV}$ transition. Thus, the stripe correlation starts to reduce the ferromagnetic correlation across the doped hole of MST at $T<T_{s}$.
More generally, the observed triplet component of the MST due to the polarization of surrounding spins implies an important change of a minimum model for the cuprates. For example, upon integrating out the triplet states, one typically arrives at the so-called $t-J$ model by dropping many nonessential high-energy terms in the process. Our observation indicates that higher-order processes like $-\left(\sum_{\text {neighbor }} \vec{S}\right)^{2} h^{+} h$ or $-\sum_{\text {neighbor }} \vec{S} \cdot h^{+} \vec{\sigma} h$ would need to be included, with $h^{+}(h)$ denoting creation (annihilation) of doped holes and $\vec{S}$ the spin of the surrounding $\mathrm{Cu}$. Interestingly, the corresponding physical effect of such terms is a tendency to align $\mathrm{Cu}$ spin ferromagnetically near doped holes without moving the doped hole, an effect emphasized by Emery and Reiter [17]. Previously, such a tendency for the development of ferromagnetic correlations was derived only from the kinetic motion of the doped holes, instead of an effective potential.

In the next discussions, we describe the optical transitions and their corresponding temperature dependence at $\sim 2.5$, $\sim 8.7, \sim 9.7, \sim 11.3$, and $\sim 21.8 \mathrm{eV}$ (see Fig. 7 ). We start our discussion on the basic electronic band structure for undoped cuprates. It is generally accepted that the parent compound SCOC, which is similar to $\mathrm{La}_{2} \mathrm{CuO}_{4}$, is an antiferromagnetic insulator with spin of $1 / 2$ on $\mathrm{Cu}$ and with a charge-transfer type conductivity gap of $\sim 2 \mathrm{eV}$ (see Fig. 1). This fixes the energy scales of the first electron addition and removal states, consistent within the pictorial model shown in Fig. 6(a) as 
(a)

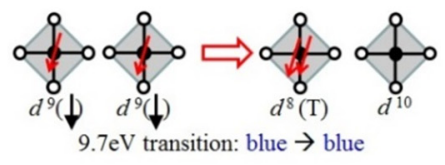

(c)

$$
\begin{aligned}
& \text { Transitions to upper Hubbard band in } \\
& \text { ferromagnetic (FM) correlation }
\end{aligned}
$$
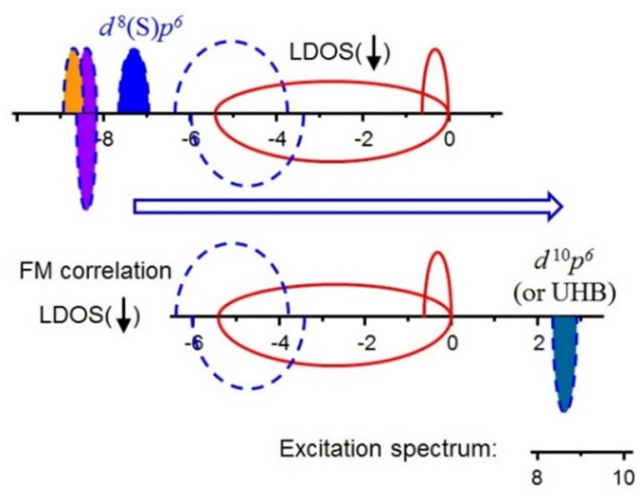

(b)

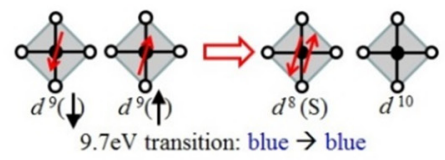

(d)

Transitions to upper Hubbard band in antiferromagnetic (AF) correlation
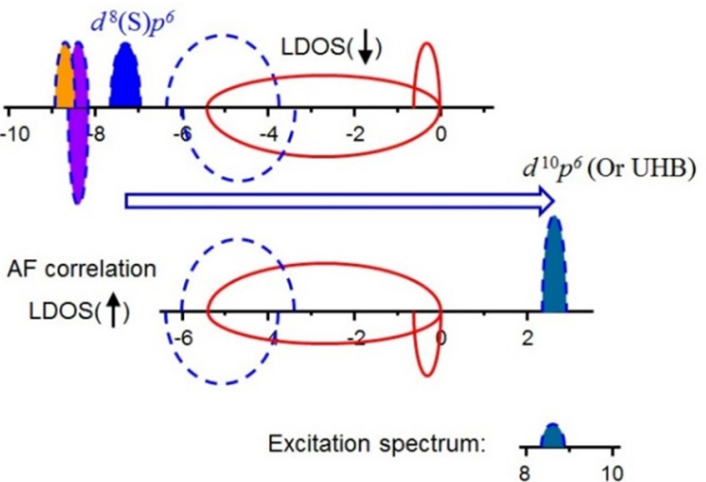

(e)

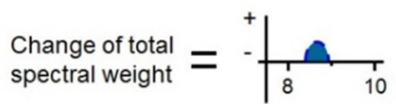

FIG. 9. Pictorial model of the electronic band structure and optical transitions at $9.7 \mathrm{eV}$. (a) Schematic electronic and spin configurations of $\mathrm{CuO}_{4}$ plaquettes. In each set of figures, the left (right) two $\mathrm{CuO}_{4}$ plaquettes represent the initial (final) state of the corresponding optical excitation. The $d^{9}$ plaquette state corresponds to the ground state of the $\mathrm{CuO}_{4}$ plaquette with a single hole. Assuming each plaquette is independent, the optical excitation energy is easily estimated from the $\mathrm{CuO}_{4}$ plaquette model. The $S$ denotes singlet configuration. (b) Excitation spectrum for transition to UHB in antiferromagnetic correlation. LDOS $(\downarrow)$ and LDOS $(\uparrow)$ denote spin down and spin up, respectively. (c) Change of total $\mathrm{SW}$ as a function of temperature is expected from this model. Note that the more complete pictorial model of electronic band structure can be seen in Fig. 5(a).

discussed later. The band width of the electron addition state is roughly $1 \mathrm{eV}$ as determined from LSDA + U calculations (see Fig. 4), and the dispersion width of the first electron removal states determined from angular resolved photoelectron spectroscopy is about $0.3 \mathrm{eV}$ or roughly twice the superexchange interaction between the local $\mathrm{Cu}$ spins [35]. This narrow electron removal structure is referred to as the ZRS, which is composed of one hole in a $\mathrm{Cu} d_{x^{2}-y^{2}}$ orbital and one hole in a linear combination of bonding $\mathrm{O} 2 p$ orbitals also with $x^{2}-y^{2}$ symmetry around the central $\mathrm{Cu}$ [Fig. 3(b)]. At higher electron removal energies, the remaining $\mathrm{O} 2 p$ orbitals form bands, which are $\sim 5 \mathrm{eV}$ wide, based on LSDA + U calculations covering an energy range to $\sim 5 \mathrm{eV}$ below the Fermi level that is fixed at the top of the valence band (see also Fig. 4). A similar feature is also obtained in the cluster calculations [see Fig. 3(b)]. Interestingly, the LSDA + U calculations show that $\mathrm{O} 2 p$ is spin polarized. At even higher electron removal energies ranging from $\sim 3.5$ to $\sim 12 \mathrm{eV}$ below the Fermi level, spin-resolved photoemission and Auger spectroscopies as well as satellite structures in photoemission spectroscopy $[27,28]$ have been found and identified as $\mathrm{Cu} d^{8}$ states, which are spread over an energy range $\sim 8 \mathrm{eV}$ due to the atomic multiplet structure resulting from the large atomic Coulomb and exchangelike interactions between the two $d$ holes (see also Fig. 3).

The $8.7 \mathrm{eV}$ feature is an optical excitation involving a hole from a ZRS to a neighboring $\mathrm{Cu}$ which ends up in a $d^{8}\left(d_{x^{2}-y^{2}}\right.$ and $\left.d_{3 z^{2}-r^{2}}\right)$ spin triplet state (Fig. 8). If we consider transitions into $d^{8}$ triplet states, this transition is expected to show a quite different temperature behavior, i.e. $\sigma_{1}$ increases as temperature decreases. Transitions into $d^{8}$ triplet states are considerably weaker in intensity involving $d_{3 z^{2}-r^{2}}$ orbitals, but more importantly, the corresponding transition to a $d^{8}$ triplet state from the doped hole plaquette that has been influenced by the transition at $9.7 \mathrm{eV}$, which is involving a $d^{8}$ singlet to the upper Hubbard band (UHB) and has an opposite temperature dependence in the $\sigma_{1}$. If, however, we could observe the transition to the $d^{8}$ triplet state, we would be using the same arguments as above but expect it to have the reversed temperature dependence.

The $9.7 \mathrm{eV}$ feature involves optical excitations of a hole from a central $\mathrm{Cu}$ in a $d^{9}\left(d_{x^{2}-y^{2}}\right)$ state to a neighboring $\mathrm{Cu}$ which ends up in a $d^{8}$ spin singlet state (Fig. 9). The temperature dependence of $\sigma_{1}$ involving the $d^{8}$ singlet and the UHB would obviously display an increase of $\sigma_{1}$ with decreasing temperature because, here, the low-temperature state would surely involve a strong antiferromagnetic alignment of the neighboring $\mathrm{Cu}$ spins. On the other hand, the ferromagnetic alignment has no contribution to this transition due to the Pauli principle.

The $11.3 \mathrm{eV}$ feature originates mainly from transitions of a hole from a central $\mathrm{Cu}$ in $d^{9}$ without the presence of a ZRS to a neighboring $\mathrm{Cu} d^{8}$ spin triplet state involving the UHB (Fig. 10). Here, we observe rather strong temperature 
(a)

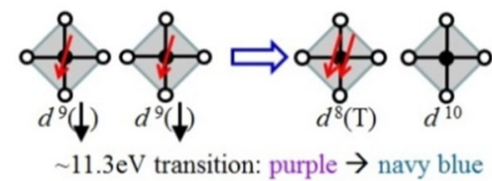

(c)

$$
\text { Transitions to upper Hubbard band in }
$$$$
\text { ferromagnetic (FM) correlation }
$$
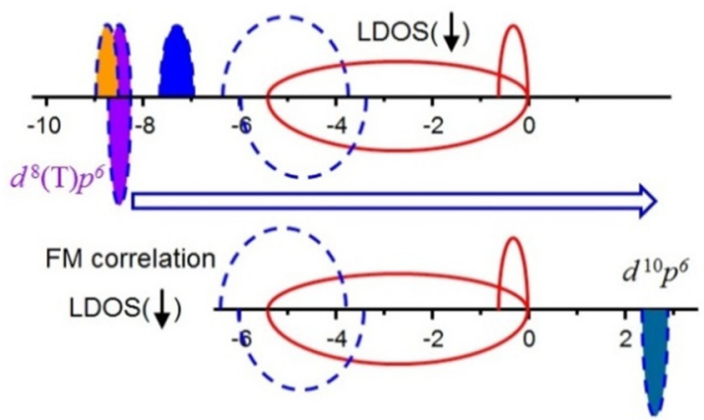

Excitation spectrum: (b)

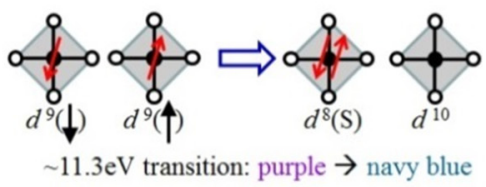

(d)

Transitions to upper Hubbard band in antiferromagnetic $(\mathrm{AF})$ correlation

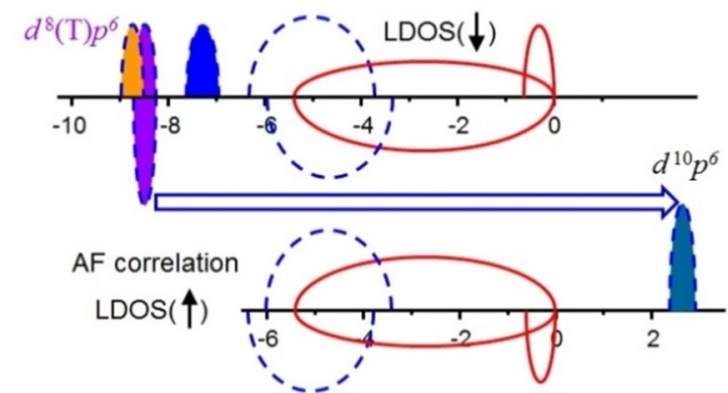

Excitation spectrum:

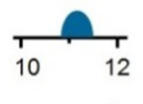

(e)

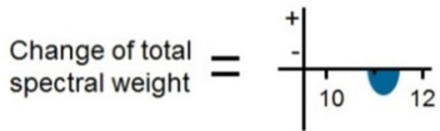

FIG. 10. Pictorial model of the electronic band structure and optical transitions at $11.3 \mathrm{eV}$. (a) Schematic electronic and spin configurations within a $\mathrm{CuO}_{4}$ plaquette for (a) transition to UHB with ferromagnetic correlations which occurs at $T_{s}$ and (b) transition to UHB in antiferromagnetic correlation which occurs at low temperatures. In each set of figures, the left (right) two $\mathrm{CuO}_{4}$ plaquettes represent the initial (final) state of the corresponding optical excitation. The $d^{9}$ plaquette state corresponds to the ground state of the $\mathrm{CuO}_{4}$ plaquette with a single hole. Assuming each plaquette is independent, the optical excitation energy is easily estimated from the $\mathrm{CuO}_{4}$ plaquette model. The $T$ and $S$ denote triplet and singlet configuration, respectively. Excitation spectrum for (c) transition in the phase with ferromagnetic correlations and (d) transitions in the phase with antiferromagnetic correlation. LDOS $(\downarrow)$ and LDOS ( $\uparrow$ ) denote spin down and spin up, respectively. (e) Change of total SW as a function of temperatures expected from this model. Note that the more complete pictorial model of electronic band structure can be seen in Fig. 5(a).

dependence, i.e. the $\sigma_{1}$ decreases as temperature decreases. This requires a starting state with the $\mathrm{Cu}$ spins parallel for the largest $\sigma_{1}$. However, the ground state is clearly one where these spins are antiparallel. The change of the SW transfer of the $11.3 \mathrm{eV}$ feature for $T \leqslant T_{s}$ is related to the stripe formation to compensate for the change of the SW transfer of the polarized MST (see also main article). The main point is that optical transitions involving the $d^{8}$ states can result in either triplet or singlet local states, which is fundamentally different from what can happen in the single band Hubbard model.

The $21.8 \mathrm{eV}$ feature corresponds to excitation of a doped hole from ZRS to the neighboring $\mathrm{O} 2 s$ core level: $\left|d^{9} \mathrm{ZRS}\right\rangle \rightarrow$ $\left|s^{1} d^{9} d^{9}\right\rangle$ [Fig. 11(a)]. With the presence of an effective exchange coupling between the $\mathrm{O} 2 s$ level with the $\mathrm{O} 2 p$ unpaired spin and indirectly with the $\mathrm{Cu}$ spin, the feature $\sim 21.8 \mathrm{eV}$, which shows a reduction at the lower energy side around $19.6 \mathrm{eV}$ and an enhancement at the higher energy side around $21.8 \mathrm{eV}$ seen in $\Delta \sigma_{1}$ [cf Figs. 1(e) and 1(f)], can be understood using the proposed MST model as illustrated in Fig. 11. Our model explains well that the optical transition from $\mathrm{O} 2 s$ to the local spin polarization results in a different sign in the change of the total SW around $21.8 \mathrm{eV}$ [Figs. 11(c) and 11(d)], while the unpolarized ZRS would be insensitive to changes of local magnetic correlations [Fig. 11(b)].

\section{CONCLUSIONS}

In conclusion, we reveal a strong mixture singlet and triplet configuration in the lightly hole-doped Zn-LSCO single crystal using high-energy optical conductivity. The doped hole is shown to induce the effective MST wave function that enhances ferromagnetic correlations between $\mathrm{Cu}$ spins near the doped holes. Our result also demonstrates a strategy and potency of high-energy optical conductivity to locally probe the interplay of magnetic correlations surrounding the doped holes in strongly correlated electron systems.

\section{ACKNOWLEDGMENTS}

We acknowledge Anthony J. Leggett and G. Baskaran for valuable discussions. This paper is supported by Singapore National Research Foundation under its Competitive Research Funding (NRF-CRP 8-2011-06), MOE-AcRF Tier-2 (MOE2015-T2-1-099), NUS-YIA, FRCs, BMBF 
(a)

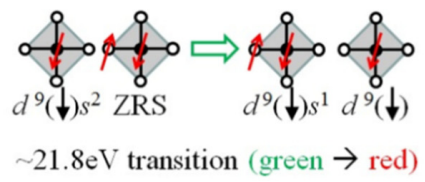

(b) Transitions to unpolarized ZRS

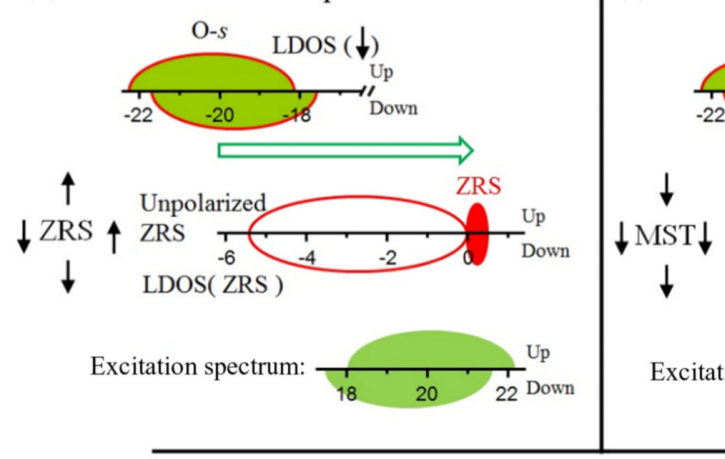

(c) Transitions to "polarized"MST

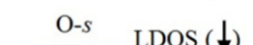

(d)

Change of total spectral-weight upon cooling $=$ the difference between MST and unpolarizedZRS

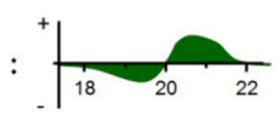

FIG. 11. Pictorial model of the electronic band structure and optical transitions at $21.8 \mathrm{eV}$. (a) Schematic hole ( $\downarrow$ ) configurations of the $21.8 \mathrm{eV}$ optical transition within two $\mathrm{CuO}_{4}$ plaquettes involving semicore $\mathrm{O} 2 s$ orbital. The calculated optical excitation energy is matched with experimental data. Note that the explanation of symbols and notations is referred to in Figs. 2 and 3. (b) An intersite optical transition from (top panel) semicore $\mathrm{O} 2 s$ to (middle panel) unpolarized ZRS and (bottom panel) corresponding excitation spectra for both spin-up and spin-down configurations based on the $\mathrm{CuO}_{4}$ plaquettes configuration in (a). This optical transition is allowed for both spin-up and spin-down configurations; the resulting excitation spectrum has equal SW in the unpolarized ZRS scheme. As a result, this transition does not change as a function of temperature because it does not depend on surrounding spin configurations. (c) An intersite optical transition from (top panel) semicore $\mathrm{O} s$ to (middle panel) spin-polarized MST and (bottom panel) corresponding excitation spectra for both spin-up and spin-down configuration. As temperature decreases, the local ferromagnetic configuration enhances. This yields to an increased SW for spin up and a decreased SW for spin down. (d) Based on MST configurations, an increased SW at the $\sim 21.8 \mathrm{eV}$ side is accompanied with a decreased SW at $\sim 20 \mathrm{eV}$, fully consistent with our experimental results [cf Figs. 1(e) and 1(f)]. Note that the O $2 s$ orbital corresponds to the $d_{x^{2}-y^{2}}$ symmetric superposition of four $\mathrm{O} 2 s$ orbitals surrounding the $\mathrm{Cu}$ and is therefore orthonormal between each site. It splits because of strong Hunds coupling and $\left|\mathrm{d}^{8} L\right\rangle$ orbital, with $L$ as a ligand hole. The $\mathrm{Cu} d$ and $\mathrm{O} p$ are both more occupied in the spin-up channel, thus lowering the energy of $\mathrm{O} 2 s$ in the spin-up channel.

through VUVFAST (05K2014) as well as DFG through $\mathrm{Ru}$ 773/4-1 and RIKEN. W.K. acknowledges support from National Natural Science Foundation of China (NSFC) \#11447601, and Ministry of Science and Technology (MOST) \#2016YFA0300500 and 2016YFA0300501. The works at
University of British Columbia are supported by NSERC, QMI, and CIfAR. The works at Tohoku University are supported by Grants-in-Aid from The Ministry of Education, Culture, Sports, Science and Technology (MEXT), Japan (No. 23340093).
[1] P. A. Lee, N. Nagaosa, and X.-G. Wen, Rev. Mod. Phys. 78, 17 (2006).

[2] F. C. Zhang and T. M. Rice, Phys. Rev. B 37, 3759 (1988).

[3] V. J. Emery, Phys. Rev. Lett. 58, 2794 (1987).

[4] M. Le Tacon, A. Sacuto, A. Georges, G. Kotliar, Y. Gallais, D. Colson, and A. Forget, Nat. Phys. 2, 537 (2006).

[5] B. Vignolle, S. M. Hayden, D. F. McMorrow, H. M. Ronnow, B. Lake, C. D. Frost, and T. G. Perring, Nat. Phys. 3, 163 (2007).

[6] Y. Li, G. Yu, M. K. Chan, V. Baledent, Y. Li, N. Barisic, X. Zhao, K. Hradil, R. A. Mole, Y. Sidis, P. Steffens, P. Bourges, and M. Greven, Nat. Phys. 8, 404 (2012).

[7] P. Kuiper, G. Kruizinga, J. Ghijsen, M. Grioni, P. J. W. Weijs, F. M. F. de Groot, G. A. Sawatzky, H. Verweij, L. F. Feiner, and H. Petersen, Phys. Rev. B 38, 6483 (1988).
[8] N. Nücker, J. Fink, J. C. Fuggle, P. J. Durham, and W. M. Temmerman, Phys. Rev. B 37, 5158 (1988).

[9] C. T. Chen, F. Sette, Y. Ma, M. S. Hybertsen, E. B. Stechel, W. M. C. Foulkes, M. Schluter, S. W. Cheong, A. S. Cooper, L. W. Rupp, Jr., B. Batlogg, Y. L. Soo, Z. H. Ming, A. Krol, and Y. H. Kao, Phys. Rev. Lett. 66, 104 (1991).

[10] E. Pellegrin, N. Nücker, J. Fink, S. L. Molodtsov, A. Gutiérrez, E. Navas, O. Strebel, Z. Hu, M. Domke, G. Kaindl, S. Uchida, Y. Nakamura, J. Markl, M. Klauda, G. Saemann-Ischenko, A. Krol, J. L. Peng, Z. Y. Li, and R. L. Greene, Phys. Rev. B 47, 3354 (1993).

[11] A. Kaminski, S. Rosenkranz, H. M. Fretwell, J. C. Campuzano, Z. Li, H. Raffy, W. G. Cullen, H. You, C. G. Olson, C. M. Varma, and H. Hochst, Nature 416, 610 (2002). 
[12] Y. Li, V. Baledent, G. Yu, N. Barisic, K. Hradil, R. A. Mole, Y. Sidis, P. Steffens, X. Zhao, P. Bourges, and M. Greven, Nature 468, 283 (2010).

[13] A. Shekhter, B. J. Ramshaw, R. Liang, W. N. Hardy, D. A. Bonn, F. F. Balakirev, R. D. McDonald, J. B. Betts, S. C. Riggs, and A. Migliori, Nature 498, 75 (2013).

[14] C. M. Varma, Phys. Rev. B 55, 14554 (1997).

[15] H. Eskes and G. A. Sawatzky, Phys. Rev. Lett. 61, 1415 (1988).

[16] B. Lau, M. Berciu, and G. A. Sawatzky, Phys. Rev. Lett. 106, 036401 (2011).

[17] V. J. Emery and G. Reiter, Phys. Rev. B 38, 4547 (1988).

[18] O. P. Sushkov, W. Xie, O. Jepsen, O. K. Andersen, and G. A. Sawatzky, Phys. Rev. B 77, 035124 (2008).

[19] A. Rusydi, R. Rauer, G. Neuber, M. Bastjan, I. Mahns, S. Müller, P. Saichu, B. Schulz, S. G. Singer, A. I. Lichtenstein, D. Qi, X. Gao, X. Yu, A. T. S. Wee, G. Stryganyuk, K. Dörr, G. A. Sawatzky, S. L. Cooper, and M. Rübhausen, Phys. Rev. B 78, 125110 (2008).

[20] M. A. Majidi, H. Su, Y. P. Feng, M. Rübhausen, and A. Rusydi, Phys. Rev. B 84, 075136 (2011).

[21] M. Matsuda, M. Fujita, and K. Yamada, Phys. Rev. B 73, 140503 (2006).

[22] L. L. Miller, X. L. Wang, S. X. Wang, C. Stassis, D. C. Johnston, J. Faber, and C. K. Loong, Phys. Rev. B 41, 1921 (1990).

[23] B. L. Henke, E. M. Gullikson, and J. C. Davis, Atom. Data Nucl. Data 54, 181 (1993).

[24] A. S. Moskvin, R. Neudert, M. Knupfer, J. Fink, and R. Hayn, Phys. Rev. B 65, 180512 (2002).
[25] S. L. Cooper, D. Reznik, A. Kotz, M. A. Karlow, R. Liu, M. V. Klein, W. C. Lee, J. Giapintzakis, D. M. Ginsberg, B. W. Veal, and A. P. Paulikas, Phys. Rev. B 47, 8233 (1993).

[26] A. Rusydi, A. Goos, S. Binder, A. Eich, K. Botril, P. Abbamonte, X. Yu, M. B. H. Breese, H. Eisaki, Y. Fujimaki, S. Uchida, N. Guerassimova, R. Treusch, J. Feldhaus, R. Reininger, M. V. Klein, and M. Rübhausen, Phys. Rev. Lett. 113, 067001 (2014).

[27] L. H. Tjeng, B. Sinkovic, N. B. Brookes, J. B. Goedkoop, R. Hesper, E. Pellegrin, F. M. F. de Groot, S. Altieri, S. L. Hulbert, E. Shekel, and G. A. Sawatzky, Phys. Rev. Lett. 78, 1126 (1997).

[28] N. B. Brookes, G. Ghiringhelli, O. Tjernberg, L. H. Tjeng, T. Mizokawa, T. W. Li, and A. A. Menovsky, Phys. Rev. Lett. 87, 237003 (2001).

[29] J. Zaanen and O. Gunnarsson, Phys. Rev. B 40, 7391 (1989).

[30] K. Machida, Phys. C 158, 192 (1989).

[31] D. Poilblanc and T. M. Rice, Phys. Rev. B 39, 9749 (1989).

[32] J. M. Tranquada, B. J. Sternlieb, J. D. Axe, Y. Nakamura, and S. Uchida, Nature 375, 561 (1995).

[33] P. Abbamonte, A. Rusydi, S. Smadici, G. D. Gu, G. A. Sawatzky, and D. L. Feng, Nat. Phys. 1, 155 (2005).

[34] A. Rusydi, W. Ku, B. Schulz, R. Rauer, I. Mahns, D. Qi, X. Gao, A. T. S. Wee, P. Abbamonte, H. Eisaki, Y. Fujimaki, S. Uchida, and M. Rübhausen, Phys. Rev. Lett. 105, 026402 (2010).

[35] K. M. Shen, F. Ronning, D. H. Lu, F. Baumberger, N. J. C. Ingle, W. S. Lee, W. Meevasana, Y. Kohsaka, M. Azuma, M. Takano, H. Takagi, and Z.-X. Shen, Science 307, 901 (2005).

[36] J. C. Slater and G. F. Koster, Phys. Rev. 94, 1498 (1954). 